inherited neurosis ; and this association is so constant that the intem. perance must be regarded, at least ultimately, as the result, rather than the cause, of the insanity.

Dipsomania has three forms-acute, periodic, and continuous. In the acute form, the patient, formerly temperate, takes suddenly to excessive drinking usually after some loss, shock, or disappointment, becomes utterly indifferent to all claims of family, business, or duty, and drinks himself to death as speedily as possible. The history of such a case is almost certain to reveal unstable nervous organisation; and probably suicide in a more sudden form has been a consequence of similar moral causes in some other member of the family.

The periodic or paroxysmal form is yet more constantly associated with some hereditary neurosis. The patient is correct and abstemious in habits, irreproachable in character, and, perhaps, of superior attain ments ; but occasionally, from anxiety, overwork, or casual indulgence, he is seized with a fierce and uncontrollable craving for stimulants. A the same time, his whole nature seems changed, and he becomes in every respect the opposite of his former self; he forgets and sacrifices everything in the gratification of this passionate craving, chooses the vilest associates, frequents the lowest haunts, resorts to cunning lies to conceal himself, lives chiefly on brandy, and is at length discovered, probably in some low pothouse, dirty, haggard, and exhausted, with the brandy by his side, for which he still craves even when the irritated stomach refuses to retain it. The attack is followed by utter loathing of alcohol and of himself for yielding to the temptation; but, after a varying number of months, the wretched experience is only too certain to be repeated.

No form of brain-disorder is more definite than this ; and, as already said, it is almost invariably associated with a hereditary tendency to in temperance, or to some form of nervous disease, which may or may no have been awakened by convivial habits, but in which habits the attacks often seem to begin.

The third form-continuous or constant dipsomania-is that where the difficulty is usually greatest in drawing the line between the vice and the disease. It is often the outcome and the result of intemperate habits, often associated with other vices, and always associated with an active form of the moral insanity, which is usually negative or merely periodic in the other types of dipsomania. It is distinguished by an amount of acute cunning, plausible hypocrisy, and fearless lying, which it is often difficult to regard as mental weakness, and difficult to de scribe except as blackguardism-so difficult, indeed, that one who has never seen this form of insanity may be pardoned for doubting its existence; but no such sceptics are ever found among those whose friends or relatives have been its victims.

I believe the mere habit of intemperance in the individual rarely produces this condition, but that it is usually a result and development of the baneful heritage entailed on their descendants by intemperate progenitors; the vice of one generation becoming the weakness of the next, liable to be evoked at any time by the parental vice, and then bringing a double curse.

The inherited tendency to intemperance may itself prompt to the habits which develope the disease; or these habits may be easily acquired in social life, the patient thoughtlessly tempting his fate ; or, again, the inherited weakness may be evoked by brain-disturbance from quite other causes, such as injury, sun-stroke, or moral shock. But, however excited, the existence of this predisposition to intem perance or nervous disorder seems the chief and essential fact in cornexion with dipsomania.

The limits of this paper allow only a word or two as to its treatment. The absolute withdrawal of alcohol, except in the very rare case where physical prostration forbids it, and the seclusion of the patient from all temptation and opportunity to indulge his habits, is of course the first and imperative step ; the next, restoration of tone, physical and moral ; and, lastly, confirmation by time of the amendment thus secured. Unfortunately, while it is easy thus to prescribe the treatment, properly to carry it out is a most difficult and trying task. The proverbial difficulty of reclaiming an inveterate drunkard is enormously increased when the drunkenness is not a habit, but a disease. While the repentance and good resolutions of the drunkard are sadly apt to be evanescent, they are at the time earnest and sincere. The dipsomaniac is scarcely ever sincere, although he can simulate sincerity most per. fectly; and scarcely ever earnest, except in accomplishing the gratification of his passion, and hating those who prevent it.

The proper treatment of dipsomania is only possible in an asylum, and seldom successful even there. At the best, it needs years of treatment and even then a relapse is always to be feared. Unhappily, asylum treatment is often impossible, since an action for damages is an unwelcome form of gratitude, and the medical and legal definitions of insanity are strangely at variance.
If the admirable endeavour to provide asylums for inebriates fortunately prove successful, they will doubtless receive dipsomaniacs as well as habitual drunkards ; but it will be almost useless to provide them without power of compulsory detention, and absurd to provide them at all without lessening the needless multitude of public-houses which so liberally supply suitable inmates.

Lastly, upon us, as physicians and alienists, a special duty rests in this matter. We see more than others of the evils of intemperance, and we are bound to tell in earnest words how dire and far-reaching is the curse it brings.

\section{ON THE ACTION OF ALCOHOL.}

\section{By JAMES ROSS, M.D., Waterfoot, near Manchester.}

As I was prevented from being present when Dr. Edmunds' paper on "The Physiological Action of Alcohol" was read at the late annual meeting of the Association, and at the subsequent discussion which took place, I hope to be allowed to make a few remarks upon the subject. It is a pity that the observations of Professor Binz could not have been reported at greater length; but the simple sentence quoted indicates the general nature of his views. Professor Binz is reported to have classified alcohol physiologically with quinine; and his opinion with regard to the action of quinine upon the white b!ood corpuscles is now very generally known. Quinine, alcohol, and other allied agents, check the movements of white blood-corpuscles, and of other minute masses of living protoplasm ; and Professor Binz, so far as I can understand his views, believes that the effects produced by these agents upon the higher organisms depend mainly upon their fundamental action upon protoplasm.

The explanation of the action of alcohol which Dr. Rutherford is reported to have given is, that it has a stimulant action upon the circulation; but, although it may be perfectly true that alcohol does, under certain circumstances, stimulate the circulation, yet the recognition of this fact is by no means an adequate explanation of its action. The forces which produce circulation are so manifold, and so complicated in their collocations, that, even if all the other effects of alcohol could be explained from this effect alone, we are almost as far as ever from a true explanation of the action of alcohol upon the higher organisms. We are led to ask, Why does alcohol stimulate the circulation? Does it stimulate the circulation under all circumstances? If not, why not? and under what circumstances does it produce no effect, or, it may be, an opposite effect? If, on the other hand, the main effects of alcohol can be deduced from its action upon simple protoplasm, we are much nearer to a true explanation of the point. The forces which produce the simple movements of protoplasm are so few and little involved, when compared with those which produce the circulation of the higher animals, that, if the effects of alcuhol upon the higher organisms could be deduced from the former action, the whole question would be resolved into simpler elements. And, indeed, living protoplasm, as represented by white blood-corpuscles and monera, is so similar to dead proteinaceous compounds, that we may entertain the hope that the action of alcohol and similar agents upon the former may, at no distant date, be resolved into their ordinary chemical action upon albuminous compounds. But, however this may be, it is perfectly certain that, if the main effects produced by alcohol upon the higher organisms can be deduced from its action upon simple protoplasm, this affords a much better explanation than if the effects were deduced from the special action of alcohol upon the circulation or upon the nervous system. Starting from the fact that alcohol checks the movements of white blood-corpuscles, let us see what are the implications. If these movements are checked, it must be because the molecules of the protoplasm have assumed relatively fixed positions-are more near than before to a condition of equilibrium. If the molecules had become very fixed, the protoplasm would be solid ; but, although this does not take place, it is clear that, if the molecules are relatively more fixed than before, the protoplasm will be correspondingly more solid. And, if the protoplasm is rendered in any degree more solid, this will retard the exchange of material which ends in growth, and the detachment of material which ends in multiplication. If, then, we carry these conceptions with us into the higher organisms, it is evident that alcohol will tend to check the growth and multiplication of protoplasm over the body, and to promote the comparatively fixed arrangement of material which constitutes structure. I do not mean to assert that alcohol tends to develope a higher degree of structure than the organism has already attained to ; but it tends to fix and consolidate the structure as it exists at the time. Briefly expressed, then, the 
effect of alcohol upon the higher organisms is to check growth and to consolidate structure.

Before, however, applying this hypothesis to the explanation of the various phenomena of the action of alcohol, we must glance rapidly at the relation which subsists between the structure and growth of a healthy organism. It is in this direction that we shall find, in my opinion, the key to the solution of the whole question. Every student of biology knows that, in living beings, aggregation cannot proceed to any great extent without a corresponding formation of structure. But, although structure is necessary to growth, it is equally true that, if the structure become too rigid and fixed, growth is checked. During the development of the individual, the forces which end in growth are very active; the structure is yielding and plastic, and becomes readily adapted to the new changes which growth necessitates; hence aggregation proceeds. But, as the individual is approaching maturity, the structure is becoming more and more fixed and unyielding; hence fur. ther aggregation is arrested. And the subsequent history of the individual from the adult condition to old age teaches the same lesson with regard to the relation subsisting between structure and growth; the structure is becoming year by year more consolidated, and the powers of growth year by year less active.

But, if it be true that excess of structure checks growth, it is no less true that excessive activity of growth breaks down structure. This truth is exemplified by such tissues as pus and cancer, which possess great activity of growtin, but which not only are not possessed of any real structure themselves, but which also produce the disappearance of large portions of the structure of the body remote from the first locality in which these tissues are formed. And what is the condition we have in acute disease? There is a very rapid growth of protoplasm or bioplasm all over the body. Dr. Beale was the first, so far as I know, to make this observation; and it is one of the most interesting and instructive observations made in recent years; nor do I think that it has yet received the attention it deserves. As a confirmation of this observation, I injected strong liquor ammonix into the thigh of a frog, and examine $\{$ four days afterwards the circulation of the mesentery. The blood-vessels were crowded with white blood-corpuscles, which could be seen sprouting in large numbers from the walls of the vessels. I am under the mark if I say that there were four times as many white blood-corpuscles under the field of the microscope in the mesentery of this frog, as there were in the mesentery of a healthy frog. If it be considered that the mesentery has no special relations with the thigh, to make us believe that these corpuscles accumulated in this locality more than they did in various other parts of the body, it will become apparent that such a multiplication of the actively growing cells of the body could only take place by the breaking down of part of its structure. If the semi-fluid substance which constitutes protoplasm had so much increased, the semi-solid substance which constitutes intercellular substance, and which represents structure, must have decreased in almost a corresponding degree. And this is one of the main sources of the increased temperature in acute disease. This is not the place to elaborate a theory of fever; but I may mention two statements usually made with regard to the sources of animal heat, which, although correct in the main, yet require careful limitation. It is said that the source of animal heat is a process of oxidation. It is perfectly true that, on its ultimate analysis, animal heat depends upon oxidation; but the quantity and distribution of the heat of an animal at a particular time may be in great measure independent of oxidation. After death from some diseases, the temperature rises and maintains itself for several hours some degrees beyond the highest fever-heat, and yet oxidation and mechanical action have almost entirely ceased. The other statement is, that the growth of plants is a process by which force is made potential, and that of animals a process by which it is rendered active. Passing over the fact that circumstances are known with regard to vegetable growth which indicate that a small amount of force is set free, we may notice the obverse fact, that a considerable amount of force must be rendered latent during the development of an animal. Force must be expended in producing organisation, and this force must be potential in the organised tissue. A considerable mass of indirect evidence might be addaced to show that organisation entails expenditure over and above that of the actions of organised tissues. I can only, however, mention one or two considerations which tend to show this. It is difficult to adduce proof that forces are expended in organising the statical tissues, such as bone and tendon; but it is evident that they must have been expended in the development of the dynamical tissues, such as nerve and muscle, and the tissues which carry on active nutrition and secretion. If an individual perform at a certain time an action unconsciously and with little effort, which at a previous time cost care and thought and labour, it is because the nerve-current passes through well defined channels, without spreading laterally and disturbing others, and thus brings into play certain groups of muscles without others not engaged in the action being affected. And this only takes place because the molecules of the tissues concerned in producing the action have become arranged into a more definite and coherent order than they possessed previously. In assuming this order, force must have been expended ; and these molecules can no more fall into a less definite and more incoherent order without giving out force, than can a brick fall from a wall to the ground without giving out heat-vibrations. There is no reason to believe that, when organised tissue becomes less organised, this is necessarily and in all cases an oxidation-process. Breaking down of structure is, in my opinion, the source of the excess of temperature in acute disease; and it is in great measure independent of oxidation. But I must now return to the action of alcohol upon the organism.

I have already said that alcohol not only tends to check growth, but that it must also tend by the same action to consolidate structure. Suppose that alcohol is given to a young animal in relatively large and continued doses, if this theory be correct, it ought to check the animal's growth and to fix prematurely its structure; and this is found to be the case. When dog-fanciers wish to produce a small pet dog, I am told that a certain amount of gin is given daily to the animal from its birth; and this proceeding is effectual in producing a dog much smaller than the parents from which it is derived, and also smaller than the other dogs of the same litter that have not been treated with gin. I was once told by a medical man that he kept a prematurely born child alive the first three weeks of its life upon nothing but whisky toddy. The child is now seven years of age, and a puny little idiot; but whether this is an effect of the whisky toddy or not, I will not venture to decide. I have, however, seen other instances of dwarfing effects upon individuals, which, I strongly suspect, were caused by the too copious imbibition of alcohol when children. There are other children, however, who are much benefited by a certain amount of alcohol, principally in the shape of wine. These are the children of parents who have a tubercular tendency. In such children, the powers of growth are very active; but the structure is deficient. A very slight irritation causes the epithelial surfaces to proliferate; and amongst their internal tissues there is a relatively large amount of that loose little organised tissue called adenoid; and these are indications of an unstable condition of the structure of the body. If such a child have a slight surfaceirritation, the neighbouring glands swell ; and subsequently the child becomes pale and thin, but grows rapidly in height. He is said to be growing beyond his strength. A moderate amount of alcohol will tend to check this growth and to consolidate the structure, and will, of course, be advantageous. I think it was Dr. Wilks who first drew special attention to the beneficial effects of a moderate allowance of wine in such cases. The beneficial effect of alcohol in the treatment of acute disease is readily explicable upon this theory. When the excessive growth of the protoplasm is checked, a further breaking down of structure is prevented. The potential force of the organised tissue is not converted into actual heat-vibrations, and consequently the tem. perature will fall.

The effects produced by abuse of alcohol as an article of diet are also explicable upon this hypothesis. The diseases to which habitual drinkers are liable may briefly be summed up as premature decay of all the powers of life, fatty degeneration, rigidity of arteries from calcification, and consequent diseases of the circulation. All of these are examples of excessive consolidation of structure along with deficient powers of growth, or the results which follow from such a condition.

I can only allude in very brief terms to the special influence which alcohol has upon the nervous system. It depends, in all probability; upon its action upon protoplasm in general, combined with its degree of diffusibility. But what of the stimulant effect of alcohol ? The stimulant action is partly delusive, partly real. In so far as it is real, it must depend upon a certain amount of nervous energy being set free. I have already said that, during the development of structure, a certain amount of force is rendered latent; but this is not the case with the consolidation of structure which alcohol produces. In the former instance, the organic material is probably raised to a higher isomeric form ; while in the latter it is equally probable that it falls into a lower. If the protoplasm, after the addition of alcohol, become in any degree more solid, without becoming more complex in chemical constitution, this must be, in the language of Mr. Herbert Spencer, an integration of matter; and there must be a concomitant dissipation of motion. When, therefore, alcohol acts upon the soft nervous tissue, it renders it slightly more solid, similar to the action it has upon other protoplasm; and along with this action there must be a concomitant quantity of nervous energy set free. It is this diffused effect which goes by the name of the stimulant action of the drug.

The degree of diffusibility of alcohol will help us to explain several 
of its special effects. Its degree of diffusibility will enable it to come in contact with nervous tissue sooner than the vegeto-alkalies, and not so soon as the anrsthetics; while in its action it holds an intermediate position between these agents. The degree of diffusibility also explains why alcohol affects the higher brain-centres first. The delicate structure of these centres, which contain comparatively little dense tissue, is soon permeated by the drug; and hence the intellect and moral nature suffer first. But I must refer the reader to the able remarks made by Mr. Herbert Spencer on this point in the appendix to the first volume of the second edition of his Psychology.

\section{THE GERM-THEORY OF DISEASE APPLIED TO THE EXPLANATION OF THE PHENOMENA OF IDIOPATHIC FEVER.*}

\section{By T. J. MA CLAGA N, M. D., Dundee.}

IDIOPATHIC fever occurs in its most typical and frequent form in the common epidemic diseases of this country. These diseases are contagious, and are due to the entrance into the system, from without, of the poison which gives rise to them.

That contagion is particulate may be accepted as proved. That it is organised is equally certain. The facts of infection, and the fact that the contagium-particles are reproduced to an enormous extent within the system, leave no room for doubt on that point. Being organised, it must be animal or vegetable.

The study of the nature and mode of action of contagion is an old one. Hitherto the subject has been looked at chiefly from an etiological point of view, and much valuable knowledge has thereby been obtained regarding both the nature of contagion and the causation of disease. It seems to me that the subject may also be studied with advantage from the pathological side, and that a consideration of the results likely to follow the propagation in the system of millions of minute organisms may throw some light on the mode of production of those phenomena which are common to the contagious fevers. A careful study of the question from this point of view has led me to the conclusion, that there is not a little fallacy in the modern views of the causation of idiopathic fever, and that the phenomena which constitute that condition are not so much the result of deranged action of the tissues, as they are the necessary consequence of the propagation in the system of minute animal organisms.

An organism which is too minute for ocular examination has its place in nature defined by its action on its environment. The action of an animal organism on its environment consists in the consumption of oxygen, nitrogen, and water, and the giving off of carbonic acid. It shall now be my endeavour to show that the propagation in the system of such an organism is competent to the production of all the phenomena of idiopathic fever. The essential phenomena of this condition are :I. Wasting of the tissues, especially the nitrogenous. 2. Increased consumption of water. 3. Preternatural heat. 4. Increased frequency of the cardiac action. 5. Increased frequency of respiration.

It is not my intention to criticise the views at present held as to the mode of production of these phenomena; I wish only to explain my own belief that each of them is a necessary result of the mere propagation in the system of an animal organism.

I. Wasting of the Nitrogenous Tissues. This must be due to one of two causes-diminished nutrition, or increased disintegration. In fever, it is believed to be due to the latter cause ; and on this belief is founded the generally accepted theory of Virchow. That there is increased metamorphosis of the tissues is unquestionable (as will be explained afterwards), but that this increase is not adequate to the production of all the phenomena of fever is equally certain. The chief agency in the loss of bulk which takes place in fever I believe to be diminished nutrition, a defective supply of the materials necessary to the formation of tissue. How is this produced? The one cause to which, directly or indirectly, are to be ascribed all the phenomena of idiopathic fever, is the contagium. This we believe to be an animal organism; as such, it consumes nitrogen. Whence does it get it ? Let us trace the nitrogen from its entrance into, to its exit from, the body; note the various changes through which it goes, and see at what point it would most readily be appropriated by the contagium-particles. The nitrogen is taken with the food, and first appears in the circulation in the form of what Voit calls circulating or store albumen ; in this form it passes to the tissues, which, in the performance of their usual nutritive action,

* Read before the Medical Section at the Annual Meeting of the British Medical Association in London, August 1873 . lay hold of the nitrogen thus conveyed to them, and incorporate it into their substance; the nitrogen thus passes into the form of what Voit has called fixed or organ-albumen; in this form it remains so long a; required, and then again passes into the circulation as circuluting albumen, in which state it is conveyed to the liver and other glands, where it is transformed into urea, and, as such, is eliminated by the kidneys. Now, at what point in this changing course is the contagiutn most likely to lay hold of the nitrogen? Clearly at that at which it would most readily be given up to it. There are four conditions in which the nitrogen has entered into such definite combinations that it is not likely to quit them at the call of the contagium; $(a)$ when it exists in the form of what I shall call constructive circulating albumen; (b) when it is fixed in the tissues ; $(c)$ when it is in the form of retrogressive circulating albumen; $(d)$ and when it has been formed into urea. There are three points at which it is in a transition state, passing from one combination to another, and is in no sense fixed; $(a)$ whe! passing from the circulating to the organ albumen; (b) when passing back from this form to that of retrogressive albumen ; and $(c)$ when it is being transformed into urea. It is at one of these three points, when the nitrogen is in a transition state and is free to enter into a new com. bination, that it is most likely to be appropriated by the contagium. At which of the three does such appropriation take place? So far as the facilities for obtaining the nitrogen are concerned, there is not much to be said in favour of any one of them ; the nitrogen is eq:ailly unstab!e in all. Much, it seems to me, must depend on the actual condition in which the nitrogen is presented to the contagium. It is evident that the state of the nitrogen, as it passes from the constructive to the organ albumen, must be very different from that in which it passes back fiom the organ albumen to the circulation; and, also, from that in which it passes from the condition of retrogressive store albumen to the form of urea. In the first case, it presents itself in a form suitable for the formation of tissue ; in the two others, it is unfit for such a purpose, and is destined only for excretion. The only time, then, at which the nitrogen presents itself in the system in a form at once unstable and fit for forming tissue, is when it is undergoing the transition which results in the formation of organ albumen from the constructive sto:e albumen. Now, it needs no elaborate argument to show that the action which results in the formation of the protoplasm of the contagium-particles, bears a much closer analogy to those constructive changes which result in the formation of the tissues, than it does to the retrograde changes which accompany their disintegration and result in the formation of urea. The formation of the tissues, and the formation of the protoplasm of the contagium particles are both constructive steps; both result in the formation of animal matter; and it is but reasonable to suppose that the only source whence the tissues can get the nitrogen which t'rey require in a suitable form, is also the most likely source of that whic's the contagium-particles require for the formation of their protoplasin. For these reasons, I believe that it is when the constructive sto:e albumen is undergoing the changes necessary to the formation from it of the organ albumen, that the nitrogen is appropriated by the contagi:umparticles. Such appropriation is the main cause of the wasting of the nitrogenous tissues. They waste because they are deprived of the nitrogen necessary to their formation. But here an apparent difficulty presents itself. Observation has shown that the amount of nitrogen eliminated from the system bears a direct relation to the quantity con: sumed, and is little, if at all, influenced by any other agency. Now, if the tissues are deprived of nitrogen by the contagium-particles in the manner indicated, how comes it that the amount of urea eliminated is, as a rule, much above the normal standard? It happens thus. The contagium-particles pervade the whole body; they take their nitrogen from the constructive albumen at the moment at which it is being yielded up to the tissues; it is in the tissues, therefore, that their protoplasm is formed. Now, the action which leads to the formation of this is identical with that which results in the formation of the normal tissues, and produces exactly the same effect as would be produced by a corresponding increase in the normal demands of these tissues. The effect of the propagation of the contagium-particles in the tissues, therefore, is the same as that which would result from excessive tissue-action. But tissue-action consists of a retrograde as well as a constructive change; these actions go on simultaneously, and neither can be said to precede the other, any more than the passage of a saline fluid through a membrane precedes the passage of an albuminous fluid situate on the other side. The moment at which the constructive albumen is undergoing the change which normally results in the formation of organ-albumen, is that at which its nitrogen is taken up by the contagium-particles. The simultaneous action by which the worn-out organ-albumen is transformed into ret:ogressive is equally far advanced. The contagium-particles ste? in to deprive the tissues of much of what they require for the furin- 